\title{
Modelado e Identificación de un Sistema Electromecánico y Diseño del Control PID para Gobernar Inalámbricamente el Desplazamiento de un Objeto Móvil
}

\section{Modeling and Identification of an Electromechanical System and PID Control Design to Wirelessly Govern the Movement of one Mobile Object}

V. Srdanovic ${ }^{1,2}$, O. I. Botero Henao ${ }^{1,3}$ y S. Hernando Ruiz Obando ${ }^{1,4}$

Recibido: 30 de octubre 2016

Aceptado: 5 de junio 2017

\section{Resumen}

En este artículo estamos presentando el proceso de diseño del control de velocidad para un sistema electromecánico, específicamente para un motor de corriente continua con imán permanente. Los resultados obtenidos son relevantes para el desarrollo del sistema de control de un objeto móvil, con el objetivo de gobernar su movimiento inalámbricamente. La metodología aplicada incluye el modelado matemático, la identificación del motor de corriente continua y el diseño del control de velocidad correspondiente. Se presentó el modelo matemático, según las leyes que describen los sistemas electromecánicos y la estipulación numérica de los parámetros que caracterizan el motor. También se realizó otro método para obtener la función de transferencia del motor, conocido como identificación de un sistema físico, aplicando la técnica experimental y computacional. En el proceso de verificación de las

1 Departamento de Electrónica, Facultad de Ingeniería, Institución Universitaria Pascual Bravo, Medellín, Colombia

2 vesna.srdanovic@pascualbravo.edu.co

3oscar.botero@pascualbravo.edu.co

4 sergio.obando@pascualbravo.edu.co 
funciones de transferencia y el diseño del sistema de control se utilizó el software de Matlab y sus respectivas herramientas como Simulink, Simscape y SISO Tool. Finalmente los controladores diseñados se usaran para desarrollar un sistema de control de desplazamiento de un objeto móvil, según una trayectoria previamente especificada. Para la comunicación inalámbrica se seleccionó la tecnología ZigBee, los pequeños radio transreceptores denominados módulos XBee, adecuados para comunicación inalámbrica a corto alcance.

Palabras clave: Modelado del motor DC, Control PID, Tecnología Arduino, Transmisión inalámbrica de datos, Protocolo ZigBee.

\section{Abstract}

In this article we are presenting the process of the speed control designing for an electromechanical system, specifically for a DC motor with permanent magnet. The results obtained are relevant for the development of the control system of one mobile object, with the aim of governing its movement wirelessly. The applied methodology includes mathematical modeling, the identification of the DC motor and the design of the corresponding speed control. The mathematical model was presented, according to the laws that describe the electromechanical systems and the numerical stipulation of the parameters that characterize the motor. Also another method was used to obtain the motor transfer function, known as identification of a physical system, applying the experimental and computational technique. In the verification process of the transfer functions and the design of the control system we used the Matlab software and its respective tools like Simulink, Simscape and SISO Tool. Finally the designed controllers will be used to develop a control system for displacement of a mobile object, according to a previously specified trajectory. For wireless communication, ZigBee technology was selected, the small radio transceivers called XBee modules, suitable for short-range wireless communication.

Keywords: Modeling of DC motor, PID control, Arduino technology, wireless data transmission, ZigBee protocol.

\section{Introducción}

Dara el desarrollo e implementación de un sistema de control de procesos físicos, son fundamentales el análisis y el modelado de los sistemas, el diseño de los controladores y la verificación de los resultados obtenidos utilizando las herramientas computacionales. Con el objetivo de desarrollar un sistema para gobernar inalámbricamente el movimiento de un objeto móvil, se realizó un análisis de las fuentes de información existentes: textos escritos en los libros de la ingeniería de control ${ }^{[1][5]}$, trabajos académico escritos ${ }^{[6]-[7]}$, los materiales de soporte técnico ofrecidos por los fabricantes ${ }^{[8][10]}$, etc.. Para desarrollo de sistema de control requerido fueron realizados: el mode- 
lado de motor de corriente continua utilizando la técnica analítica (modelo matemático) y la técnica experimental (identificación del motor), el diseño del controlador utilizando las herramientas disponibles en el software de Matlab y finalmente la verificación de los resultados obtenidos. El sistema de control diseñado se utilizará para implementar el control de movimiento de un objeto móvil, con necesidad específica de desplazarlo según una trayectoria arbitrariamente seleccionada.

\section{Modelado del motor de corriente continua}

\subsection{Presentación del modelo matemático}

En el campo de la ingeniería de control, el proceso de modelado matemático de sistemas físicos es muy importante; se trata de caracterizar el comportamiento dinámico del sistema por medio de un conjunto de expresiones matemáticas. Debido a la complejidad de los sistemas, es necesario introducir ciertas suposiciones, así que el modelo matemático queda más sencillo, pero que también representa la dinámica del sistema de una manera bas-

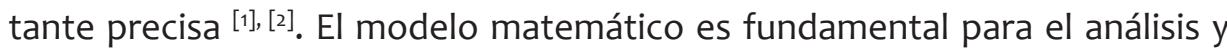
el diseño de los sistemas de control (para observar la estabilidad y exactitud del sistema, realizar el diseño de controladores, analizar la respuesta del sistema frente a las diferentes señales de prueba, etc.) ${ }^{[3]-[5]}$. También es de vital importancia el uso de las herramientas computacionales como el software Matlab, que involucra sus cajas de soporte (Toolbox) y módulos especializados como el Simulink y Simscape.

El motor seleccionado para el desarrollo es de corriente continua con imán permanente, de relativamente pequeño tamaño. Estos tipos de motores son frecuentemente utilizados en las aplicaciones de robótica, mecatrónica, juguetes electrónicos controlados remotamente, etc..

A continuación se muestra la técnica analítica para obtener el modelo matemático del motor seleccionado. En la Fig.1 se representa el esquema del sistema electromecánico del motor de corriente continua con imán permanente $[3],[1],[12]$. El estator del motor, donde se encuentra el imán permanente, es representado por medio de un par de polos magnéticos $(\mathrm{N}-\mathrm{S})$ con un flujo magnético constante ( $\Phi=$ const.). La armadura del motor es representada por medio de un circuito eléctrico donde: $v_{a}$ es el voltaje de corriente conti- 
nua de alimentación del motor, $i_{a}$ es la corriente que circula por los devanados ubicados en la armadura del motor, $R_{a}$ es la resistencia del conductor de cobre de los devanados, $L_{a}$ es la inductancia de los devanados y $e_{b}$ es el voltaje inducido en los devanados (denominado la fuerza contra-electromotriz). La parte mecánica del motor está representada por medio de un momento de inercia de armadura $(J)$ y el coeficiente de fricción viscosa $(b)$. Se supone que la inercia del engranaje del motor es despreciable y que no se encuentra aplicada la carga mecánica externa en el eje del motor.

El motor energizado con el voltaje $v_{a}$, produce el torque del motor $\left(T_{m}\right)$ que acciona el giro de su eje. Debido a que los devanados de la armadura giran dentro del campo magnético fijo, se induce el voltaje $e_{b}$ en los devanados de la armadura, opuesto al voltaje suministrado $\left(v_{a}\right)$.

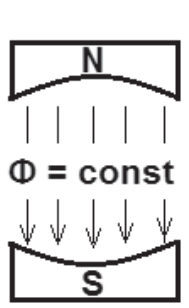

Estátor



Circuito eléctrico de armadura

Figura 1. PRESENTACIÓN ESQUEMÁtICA DE SISTEMA ELECTROMECÁNICO DE UN MOTOR DE CORRIENTE CONTINUA CON IMÁN PERMANENTE.

Las ecuaciones fundamentales que caracterizan el sistema electromecánico del motor de corriente continua con imán permanente, son presentadas a continuación. La ecuación (1) caracteriza el circuito eléctrico de la armadura y está descrita con base en las leyes fundamentales de los circuitos eléctricos. La ecuación (2) caracteriza el movimiento giratorio de la armadura en la presencia del torque del motor $\left(T_{m}\right)$ y el torque de fricción viscosa. El torque generado por la fricción viscosa es igual al producto de la velocidad de giro de la armadura $(w)$ y el coeficiente de fricción viscosa $(b)$ y se opone al torque del motor. La ecuación (3) presenta la relación proporcional entre el tor- 
que del motor $\left(T_{m}\right)$ y la corriente de la armadura $\left(i_{a}\right)$, donde la constante de proporcionalidad es $K_{t}$ (constante de torque electromagnético). La ecuación (4) expresa la relación proporcional entre el voltaje inducido en armadura $\left(e_{b}\right)$ y la velocidad angular de la armadura $(w)$, donde la constante de proporcionalidad es $K_{b}$ (constante de fuerza contra-electromotriz) ${ }^{[3]}$.

$$
\begin{aligned}
& v_{a}=\left(R_{a} * i_{a}\right)+\left(L_{a} * \frac{d i_{a}}{d t}\right)+e_{b} \\
& J * \frac{d w}{d t}=T_{m}-(b * w) \\
& T_{m}=K_{t} * i_{a} \\
& e_{b}=K_{b} * w
\end{aligned}
$$

Las constantes de torque del motor $K_{t}$ y de fuerza contra-electromotriz $K_{b}$, son de igual magnitud, según el principio de conservación de la energía, sin embargo tienen diferentes unidades (ecuación (5)) ${ }^{[4]}$.

$$
K_{t}\left[\frac{N m}{A}\right]=K_{b}\left[\frac{V}{r a d / s e g}\right]
$$

En la Tabla 1 se presentan los parámetros que caracterizan el modelo matemático del motor de corriente continua con sus respectivas unidades.

TABLA 1. NOMENCLATURA DE LOS PARÁMETROS DEL MOTOR DE CORRIENTE CONTINUA CON IMÁN PERMANENTE.

\begin{tabular}{|l|l|}
\hline Parámetro & Descripción \\
\hline$R_{a}[\Omega]$ & Resistencia interna de armadura del motor \\
\hline$L_{a}[\mathrm{H}]$ & Inductancia interna de armadura del motor \\
\hline$K_{b}[\mathrm{~V} /(\mathrm{rad} / \mathrm{s})]$ & Constante de la fuerza contra-electromotriz \\
\hline$K_{t}[\mathrm{~N} \cdot \mathrm{m} / \mathrm{A}]$ & Constante de torque electromecánico \\
\hline$J[\mathrm{~kg} \cdot \mathrm{m} 2]$ & Momento de inercia de armadura \\
\hline$b[(\mathrm{~N} \cdot \mathrm{m}) /(\mathrm{rad} / \mathrm{s})]$ & Coeficiente de fricción viscosa \\
\hline
\end{tabular}


El conjunto de ecuaciones, previamente presentado, permite utilizar la técnica clásica de modelado del motor o sea representar la relación entre la entrada (voltaje de armadura) y el salida (velocidad angular) del motor. Por medio de la técnica clásica de modelado se obtiene la función de transferencia del motor (ecuación (6)), que requiere aplicar la transformada de Laplace sobre el conjunto de ecuaciones matemáticas y determinar la relación de la salida del motor (velocidad de giro) y la entrada (voltaje de armadura).

$$
\frac{W(s)}{V_{a}(s)}=\frac{K_{t}}{\left(L_{a} * s+R_{a}\right) *(J * s+b)+K_{b} * K_{t}}
$$

También se tiene la base necesaria para continuar con la aplicación de las herramientas computacionales, específicamente el software de Matlab. En la Fig.2 se presenta el modelo matemático del motor de corriente continua en Simulink, previamente desarrollado en forma analítica.

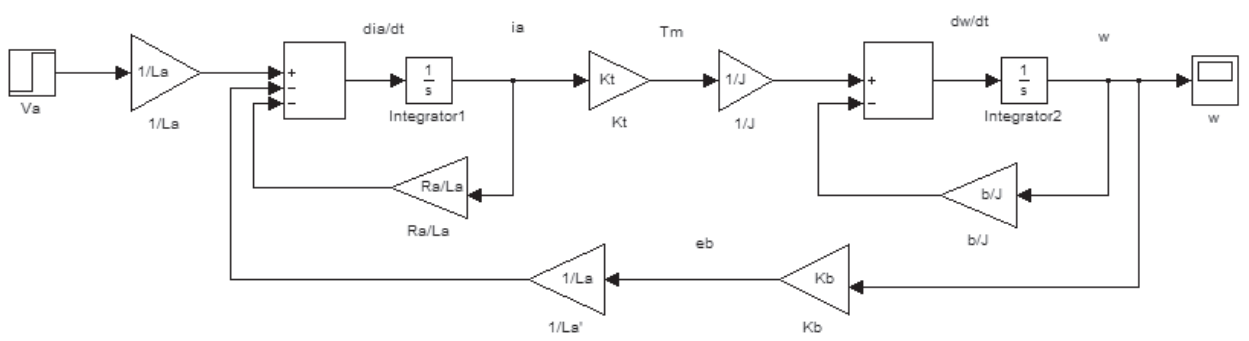

FIGURA 2. MODELO MATEMÁTICO DEL MOTOR DE CORRIENTE CONTINUA REPRESENTADO EN EL SIMULINK.

El software de Matlab tiene otro paquete, denominado Simscape, específicamente diseñado para facilitar el modelado de ciertos sistemas físicos. En la Fig.3 se observa el modelo del motor de corriente continua representado en Simscape. La ventaja de esta herramienta es que no requiere previo conjunto de ecuaciones matemáticas, ya que incluye los bloques funcionales que caracterizan el comportamiento dinámico del motor. 


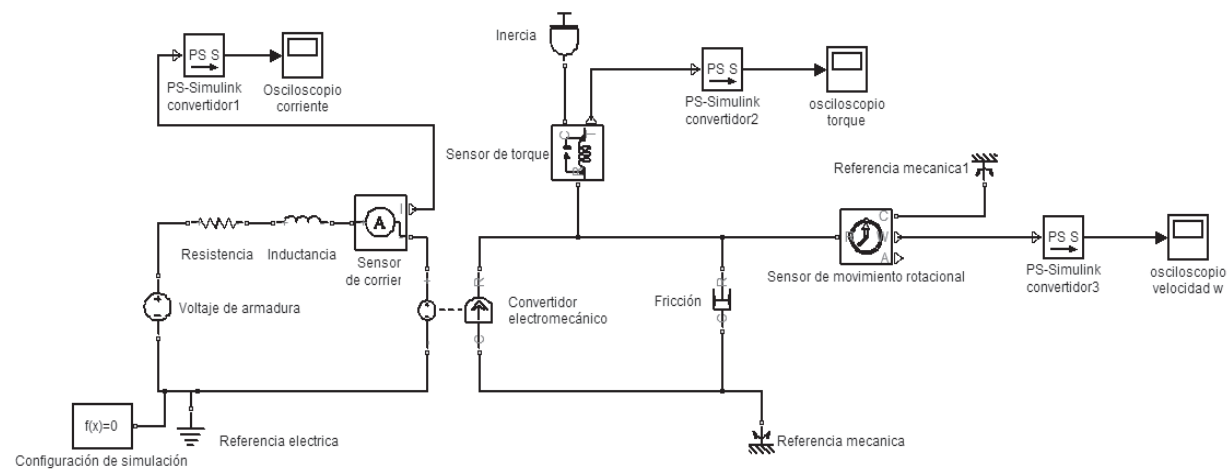

FIGURA 3. MODELO MATEMÁTICO DEL MOTOR DE CORRIENTE CONTINUA REPRESENTADO EN SIMSCAPE.

\subsection{Estimación de los parámetros del motor de corriente continúa}

Para continuar con el desarrollo del sistema de control es necesario obtener los valores numéricos de los parámetros del motor. Para este proyecto se ha seleccionado el motor de corriente continua del fabricante Pololu Robotics\&Electronics, cuya presentación física se puede observar en la Fig.4 ${ }^{[13]}$. El motor tiene incorporado un engranaje o caja reductora de velocidad, con una relación de 30:1. Muchos fabricantes implementan el sistema de engranaje, en los motores de corriente continua, para mejorar el torque del motor y reducir la velocidad de giro. Estos motores también son denominados motorreductores.



FIGURA 4. EL MOTOR DE CORRIENTE CONTINUA DEL FABRICANTE POLOLU ROBOTICS\&ELECTRONICS.

[FUENTE: HTTPS://WWW.POLOLU.COM/PRODUCT/1103] 
Los parámetros eléctricos del motor seleccionado, suministrados por el fabricante Pololu Robotics\&Electronics, se encuentran en la Tabla 2.

TABLA 2. LOS PARÁMETROS ELÉCTRICOS DEL MOTOR SUMINISTRADOS POR EL FABRICANTE.

\begin{tabular}{|l|l|}
\hline Parámetro & Valor \\
\hline Voltaje nominal & $12 \mathrm{VDC}$ \\
\hline Velocidad nominal sin carga & $350 \mathrm{RPM}$ \\
\hline Corriente nominal sin carga & $300 \mathrm{~mA}$ \\
\hline Torque máximo (rotor bloqueado) & $8 \mathrm{kgf.cm}$ \\
\hline Corriente máxima (rotor bloqueado) & $5 \mathrm{~A}$ \\
\hline
\end{tabular}

En el modelo matemático previamente presentado están identificados los parámetros que caracterizan las propiedades eléctricas y mecánicas del motor (Tabla 1). Para determinar los valores numérico de estos parámetros se utilizo la metodología de medición directa o métodos indirectos. Los valores de la resistencia de armadura $(R a)$ y de la inductancia de armadura $(L a)$ son determinados mediante la medición directa, utilizando los instrumentos: el óhmetro y el medidor RLC, respectivamente. Se realizaron varias mediciones, para diferentes posiciones del eje de motor, para promediar los valores de $\mathrm{Ra}$ y La. La constante de la fuerza contra-electromotriz $(K b)$ se estimó con base en mediciones realizadas en el régimen de operación permanente del motor. Para diferentes valores de voltaje aplicado en la armadura del motor ( $v a$ ) se realizó la medición de la corriente (ia) y se realizó la medición de velocidad de giro del eje de motor $(w)$; los instrumentos de medición utilizados fueron: voltímetro, amperímetro y tacómetro digital, respectivamente. El método indirecto utilizado para obtener el valor $K b$ fue el siguiente: de las ecuaciones (1) y (4) se despejó la constante $K b$ y se calculó la misma reemplazando los valores medidos de va, ia y w; es importante tener en cuenta que el motor en régimen permanente tiene la corriente ia=constante y por lo tanto el termino de la derivada de la corriente ia se anula en la ecuación (1). También el coeficiente de fricción viscosa (b) se obtuvo mediante el modo indirecto. De las ecuaciones (2) y (3), se despejó el coeficiente de fricción viscosa (b) y se 
calculó reemplazando los valores medidos de la corriente de armadura (ia) y de la velocidad angular $(w)$. Como en el régimen permanente del motor, la velocidad de giro $w=$ constante, se anula el término de la derivada de la velocidad angular $w$ en la ecuación (2). Por último el momento de inercia de la armadura $J$ se determinó por medio de la medición de la constante de tiempo mecánico ( $\mathrm{\tau m})$. Esta constante de tiempo es estimada como un tercio del tiempo que trascurre entre la desconexión de la alimentación del motor y su detención. El momento de inercia J es calculado como el producto de $\tau \mathrm{m}^{*} \mathrm{~b}$. En la Tabla 3 se presentan los resultados obtenidos de los valores de parámetros del motor seleccionado.

TABLA 3. LOS PARÁMETROS ESTIMADOS DEL MOTOR.

\begin{tabular}{|c|c|c|}
\hline Parámetro & Valor & Técnica aplicada \\
\hline $\begin{array}{l}\text { Ra (resistencia de } \\
\text { armadura) }\end{array}$ & $2,94[\Omega]$ & Medición directa (óhmetro) \\
\hline $\begin{array}{l}\text { La (inductancia de } \\
\text { armadura) }\end{array}$ & $2,31[\mathrm{mH}]$ & $\begin{array}{c}\text { Medición directa (medidor } \\
\text { LCR) }\end{array}$ \\
\hline $\begin{array}{l}\text { Kb (constante } \\
\text { de fuerza contra } \\
\text { electromotriz) }\end{array}$ & $0,327[\mathrm{~V} /(\mathrm{rad} / \mathrm{seg})]$ & $\begin{array}{l}\text { Estimado con base en } \\
\text { mediciones de Va, la y w. }\end{array}$ \\
\hline $\begin{array}{l}\text { Kt (constante de } \\
\text { torque del motor) }\end{array}$ & $0,327[\mathrm{~N} \cdot \mathrm{m} / \mathrm{A}]$ & $\begin{array}{l}\text { Estimado con base en } \\
\text { mediciones de Va, la y w. }\end{array}$ \\
\hline $\begin{array}{l}b \text { (coeficiente de } \\
\text { fricción viscosa) }\end{array}$ & $0,00345[\mathrm{~N} \cdot \mathrm{m} /(\mathrm{rad} / \mathrm{s})]$ & $\begin{array}{l}\text { Estimado con base en } \\
\text { mediciones de la y w. }\end{array}$ \\
\hline $\begin{array}{l}\text { J (momento de } \\
\text { inercia) }\end{array}$ & $0,0012\left[\mathrm{Kg} \cdot \mathrm{m}^{2}\right]$ & $\begin{array}{l}\text { Estimado mediante medición } \\
\text { de constante de tiempo } \\
\text { mecánica tm. }\end{array}$ \\
\hline
\end{tabular}

\subsection{Verificación del modelo matemático}

Reemplazando los valores de los parámetros previamente determinados (Tabla 3) en la función de transferencia presentada en la ecuación 6 se obtiene la ecuación (7).

$$
\frac{W(s)}{V_{a}(s)}=\frac{117965,4}{s^{2}+1275,6 * s+42243,87}
$$


El próximo paso aplicado en el desarrollo es la verificación del modelo de motor. Se utilizó el software de Matlab. En la Fig. 5 se presenta la respuesta (velocidad de giro del motor) cuando en la entrada se aplicó una señal tipo escalón de magnitud 12 (correspondiente a un voltaje nominal del motor de $12 \mathrm{~V}_{\mathrm{DC}}$ ). Se observa que la velocidad de giro del motor en estado estacionario es de 33,5 [rad/seg]. Realizando la conversión de unidades de [rad/seg] a rpm (revoluciones por minuto), la velocidad de giro obtenida de la simulación en Matlab, es 319.9 [rpm].

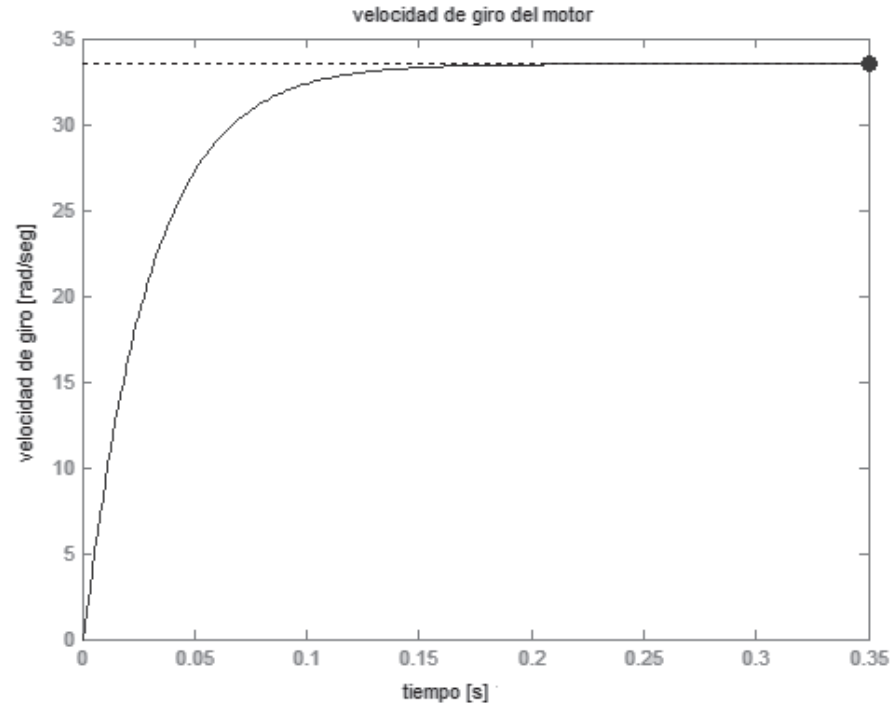

FIGURA 5. SIMULACIÓN EN MATLAB DE LA VELOCIDAD DE GIRO DEL MOTOR PARA UN VOLTAJE NOMINAL DE $12 V_{D C}$ EN LA ARMADURA.

En la Tabla 4 se observan los valores de la velocidad de giro del motor provenientes de tres fuentes. El primer valor es el dato suministrado por el fabricante. El segundo valor es el obtenido por medición directa de velocidad del motor utilizando un tacómetro digital. El tercer valor es el obtenido de la simulación en Matlab con base en el modelo matemático desarrollado previamente. Se observa que el valor medido de velocidad de giro del motor y el valor obtenido de la simulación son muy cercanos, lo que indica que el 
modelo matemático del motor desarrollado es válido para el análisis del sistema y el diseño del control.

TABLA 4. COMPARACIÓN DE VALORES DE VELOCIDAD DE GIRO DEL MOTOR PARA UN VOLTAJE NOMINAL DE $12 \mathrm{~V}_{D C}$

\begin{tabular}{|l|l|l|}
\hline Velocidad del giro de motor & Valor rpm & Valor rad/seg \\
\hline Valor suministrado por el fabricante & $350 \mathrm{rpm}$ & $36,65 \mathrm{rad} / \mathrm{seg}$ \\
\hline Valor medido con el tacómetro & $326 \mathrm{rpm}$ & $34,14 \mathrm{rad} / \mathrm{seg}$ \\
\hline Valor obtenido de la simulación & & $33,5 \mathrm{rad} / \mathrm{seg}$ \\
\hline
\end{tabular}

De la simulación obtenida en Simulink (Fig. 6) se puede confirmar: el voltaje tipo escalón de magnitud 12 aplicado en la entrada del motor genera la velocidad de giro del motor de aproximadamente $34 \mathrm{rad} / \mathrm{seg}$ como salida o respuesta del motor.



FIGURA 6. SIMULACIÓN EN SIMULINK DE LA VELOCIDAD DE GIRO DEL MOTOR PARA VOLTAJE NOMINAL DE $12 V_{D C}$ EN LA ARMADURA.

Otra simulación fue realizada en Simscape de Matlab, bajo el mismo voltaje nominal de $12 \mathrm{~V}_{D C}$ en la entrada (Fig.7). Es de interés observar que en estado permanente la corriente nominal del motor alcanza un valor muy próximo a los $300 \mathrm{~mA}$. Este mismo valor es el suministrado por el fabricante Pololu Robotics\&Electronics (Tabla 2). Es otra prueba que convalida positivamente el modelo matemático del motor previamente presentado. 




FIGURA 7. SIMULACIÓN EN SIMSCAPE DE LA CORRIENTE DEL MOTOR PARA UN VOLTAJE NOMINAL DE $12 V_{D C}$ EN LA ARMADURA.

\subsection{Identificación del Motor de Corriente Continua}

La técnica experimental es la otra forma de obtener el modelo de un sistema dinámico y se conoce como identificación del sistema. Esta técnica requiere aplicar cierta excitación y observar la respuesta del sistema. Para el caso del motor de corriente continua, el procedimiento de identificación se realizó utilizando la tarjeta de Arduino UNO (para la adquisición de los datos) y el software Matlab para su almacenamiento y desarrollo del modelo. Para interconectar el Arduino con Matlab se requiere la instalación previa de un Toolbox apropiado en Matlab. También se necesita programar la tarjeta de Arduino Uno (el código de programación esta suministrado por la corporación MathWorks) ${ }^{[4]}$.

En la Fig. 8 se observa un conjunto de dos motores acoplados, conocido como motor-generador, utilizado para proceder experimentalmente con la identificación del motor. Son dos motores de corriente continua de las mismas características (previamente descritas), acoplados eje con eje mecánicamente. El sistema motor-generador se utilizó en la siguiente manera: en el extremo del motor se aplicó un voltaje nominal de $12 \mathrm{~V}_{D C} \mathrm{y}$ en otro extremo del generador se captó la señal eléctrica (el voltaje generado). Según la ecuación (4) el voltaje inducido es proporcional a la velocidad de giro del motor-generador. 


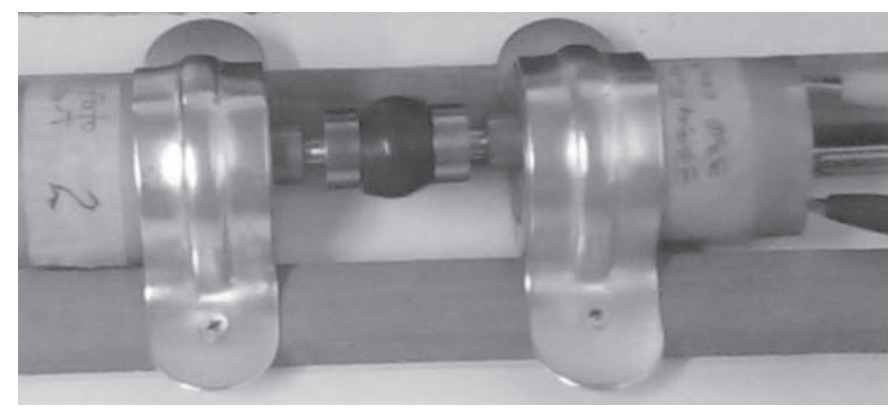

FIGURA 8. EL SISTEMA MOTOR-GENERADOR UTILIZADO PARA ADQUISICIÓN DE VOLTAJE INDUCIDO PROPORCIONAL A LA VELOCIDAD DE GIRO DEL MOTOR.

En la Fig. 9 se presentan los resultados de la adquisición de datos obtenidos del sistema motor-generador, por medio de la tarjeta de Arduino UNO y el software de Matlab. Es importante aclarar que la tarjeta de Arduino UNO no soporta la adquisición de señales de magnitud superior a $5 \mathrm{~V}_{\mathrm{DC}}$, por lo que fue necesario atenuar las señales en el orden de diez veces. Esto significa que los valores obtenidos en el proceso de adquisición, fueron de magnitud diez veces menores. En la parte superior de la Fig. 9 se observa el voltaje aplicado en la armadura del motor, que tiene la forma de un escalón de magnitud $1,2 \mathrm{~V}_{D C}$ (valor atenuado de $1,2 \mathrm{~V}_{D C}$ corresponde al voltaje nominal de $12 \mathrm{~V}_{D C}$ ). En la parte inferior de la figura se observa la respuesta del sistema motor-generador; la señal eléctrica de aproximadamente $0,9 \mathrm{~V}_{D C}$ en estado estacionario (valor atenuado de $0,9 V_{D C}$ corresponde a $9 V_{D C}$ real). Esto significa que voltaje inducido realmente fue de $9 \mathrm{~V}_{\mathrm{DC}}$.

Para verificar el resultado obtenido experimentalmente del sistema motor-generador fue necesario observar de nuevo la ecuación 4. Despejando la velocidad de giro y reemplazando los valores conocidos del voltaje inducido y de la constante de la fuerza contra-electromotriz se obtiene el valor presentado en la ecuación (8). La velocidad del motor es de 27,5[rad/seg] cuando el motor esta energizado con el voltaje nominal y está por debajo del valor de 36,65[rad/seg] suministrado por el fabricante (Tabla 4). Es algo esperado debido a las perdidas mecánicas y eléctricas. Sin embargo el proceso experimental de identificación de sistemas es importante, específicamente para los sistemas complejos cuando el modelado matemático queda muy difícil de implementar. 



FIGURA 9. EL RESULTADO DE ADQUISICIÓN DE DATOS PARA LA IDENTIFICACIÓN DEL MOTOR, OBTENIDO POR MEDIO DE LA TARJETA ARDUINO UNO Y DEL MATLAB.

$$
w=\frac{e_{b}}{K_{b}}=\frac{9 \mathrm{~V}}{0,327 \frac{\mathrm{V}}{\mathrm{rad} / \mathrm{seg}}}=27,5\left[\frac{\mathrm{rad}}{\mathrm{seg}}\right]
$$

La identificación del motor de corriente continua se desarrolla utilizando el software de Matlab, específicamente la herramienta para la Identificación de Sistemas (System Identification Tool) ${ }^{[15],[16]}$. Se requiere ingresar los datos numéricos de las señales de la entrada y de la salida del motor-generador, obtenidos en el proceso de adquisición por medio de Arduino UNO (Fig.10). Una vez ingresados los datos se especifica el método de aproximación numérica requerida para obtener finalmente la función de transferencia. En este caso se utilizó la aproximación a sistema de primer orden. 


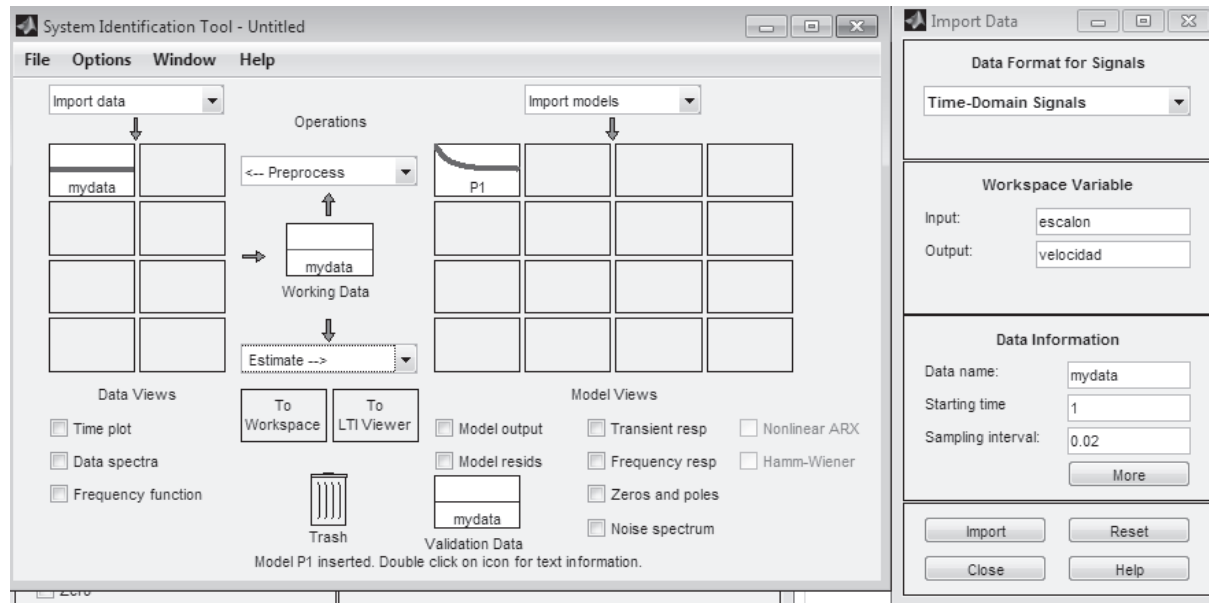

FIGURA 10. LA HERRAMIENTA PARA IDENTIFICACIÓN DE SISTEMAS FÍSICOS DEL MATLAB.

En la ecuación (9) se presenta la función de transferencia del motor de corriente continua, obtenida por medio de la herramienta de Identificación de Sistemas disponible en el software de Matlab.

$$
G(s)=\frac{0,73196}{1+0,41419 * s}
$$

Para verificar la función de transferencia del motor desarrollada experimentalmente (ecuación (9)) se utilizo el software de Matlab; se aplicó como entrada la señal de escalón de magnitud 12 y se obtuvo la respuesta presentada en la Fig. 11. Por otro lado se utilizó el osciloscopio; en la Fig. 12 se observa la señal de salida del motor-generador cuando en la entrada se aplicó el voltaje nominal de $12 \mathrm{~V}_{\mathrm{DC}}$. Los valores son relativamente cercanos: el valor de $8.8 \mathrm{~V}$ obtenido utilizando el software de Matlab y el $9,73 \mathrm{~V}$ obtenido utilizando el osciloscopio. 
Srdanovic, et al. / Modelado e Identificación de un Sistema Electromecánico y Diseño...

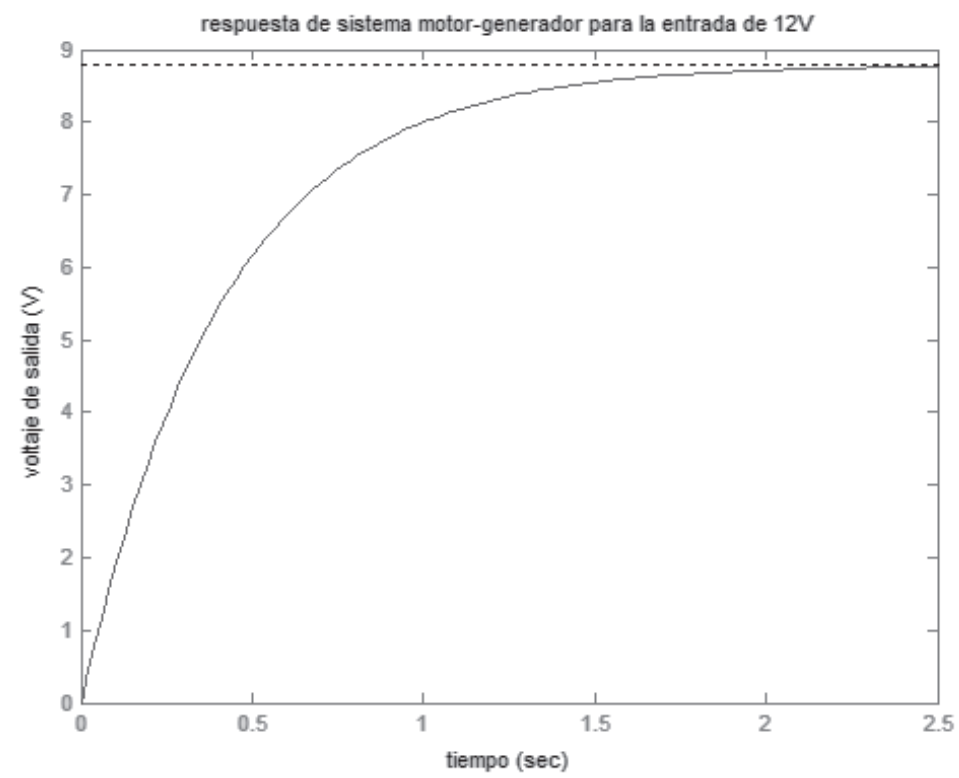

FIGURA 11. VERIFICACIÓN DE LA FUNCIÓN DE TRANSFERENCIA OBTENIDA POR IDENTIFICACIÓN DEL MOTOR EN MATLAB.

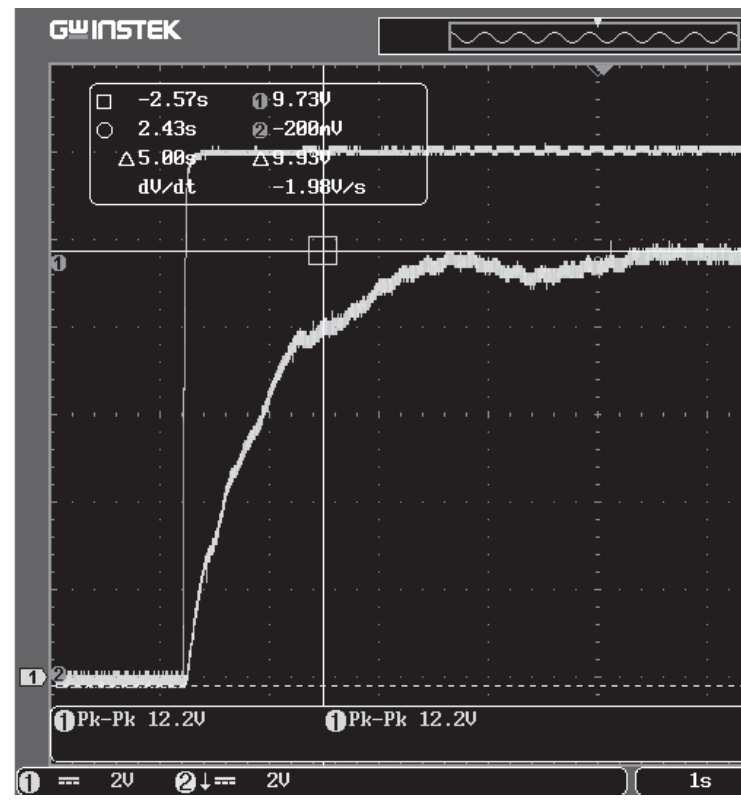

FIGURA 12. VOLTAJE EN SALIDA DE MOTOR-GENERADOR UTILIZANDO EL OSCILOSCOPIO. 


\section{Diseño de control PID}

Las funciones de transferencia obtenidas en el proceso de modelado del motor, son utilizadas para el análisis y diseño del controlador PID. La primera función de transferencia (ecuación (7)), representa el motor como un sistema de segundo orden y es más exacta, mientras que la segunda (ecuación (9)) corresponde a un sistema de primer orden y es más simple para el análisis. El diseño de controlador se realizó utilizando la herramienta de diseño de sistemas de control SISO (Single Input Single Output) Design de Matlab ${ }^{[1][[21]}$. Utilizando la función de transferencia del motor dada en la ecuación (7), se diseñó el controlador PID y la respuesta del sistema de control se presenta en la Fig. 13. El sistema con el controlador PID implementado, tiene la respuesta con las siguientes características: el error en estado estacionario se elimina (el sistema de control es preciso), la duración del régimen transitorio es de aproximadamente $5 \mathrm{~ms}$ (muy corta) y el sobre pico de la respuesta en el régimen transitorio es de $3 \%$ (bajo).

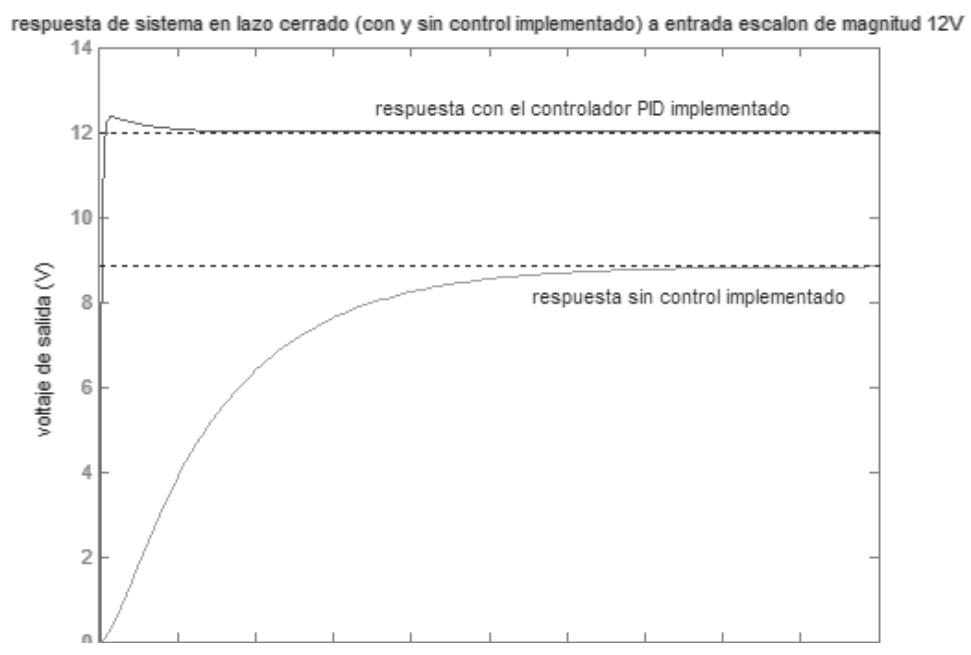

FIGURA 13. RESPUESTA DEL SISTEMA CON EL CONTROLADOR PID IMPLEMENTADO (DISEÑAdo CON BASE EN EL MODELO MATEMATICO DEL MOTOR).

También se realizó el diseño del controlador utilizando la función de transferencia de la ecuación (9). En este caso el mejor desempeño lo presenta el 
controlador PI (Proporcional - Integral). En la Fig. 14 se presenta la respuesta del sistema con el controlador PI implementado.

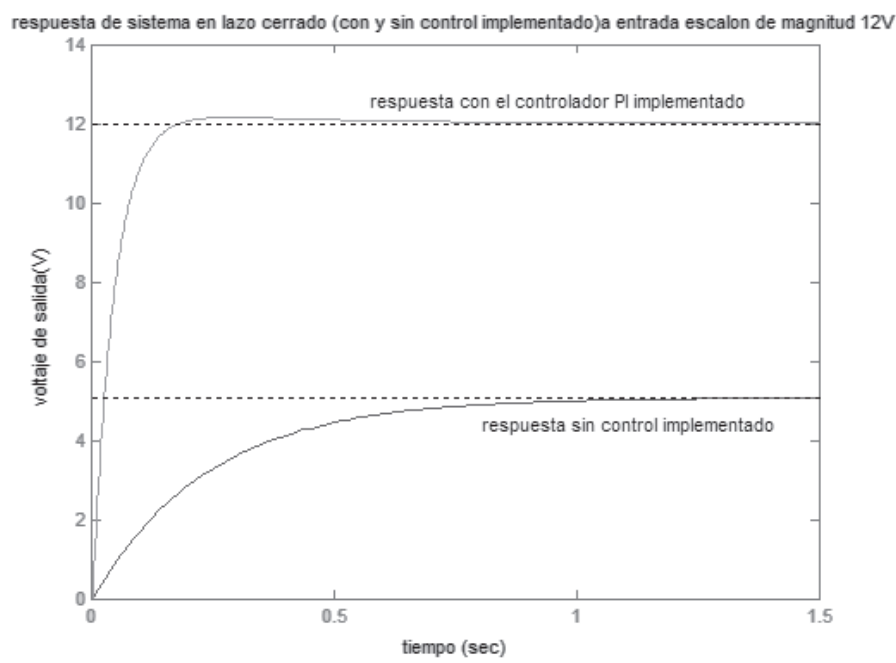

FIGURA 14. RESPUESTA DEL SISTEMA CON EL CONTROLADOR PI IMPLEMENTADO (DISEÑADO CON BASE EN LA IDENTIFICACION DEL MOTOR).

El sistema con el controlador PI implementado, tiene la respuesta con las siguientes características: el error en estado estacionario se elimina (el sistema de control es preciso), la duración del régimen transitorio es de aproximadamente 500ms (relativamente corta) y el sobre pico de la respuesta en el régimen transitorio es de $1.3 \%$ (muy bajo).

Finalmente se realizo la prueba de sistemas de control diseñados aplicando un cambio dinámico en la entrada (tren de pulsos). En la Fig. 15 se presenta la respuesta de sistema de control con el controlador PID implementado y en la Fig. 16 con el controlador PI implementado. En ambos casos los sistemas de control diseñados responden satisfactoriamente. 


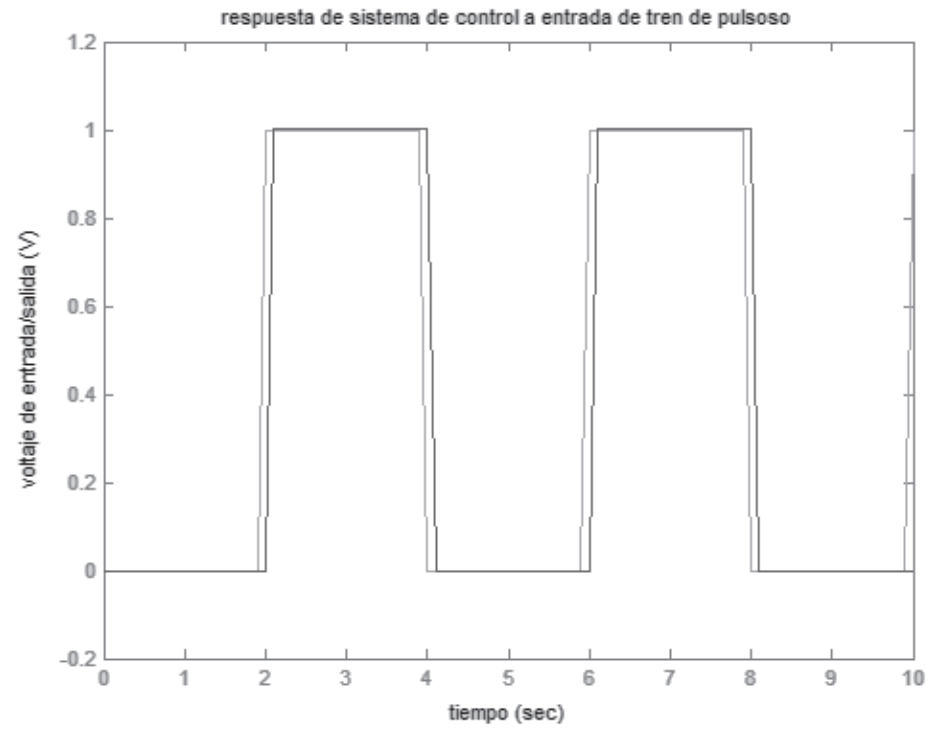

FIGURA 15. RESPUESTA DEL MOTOR GOBERNADO POR EL CONTROLADOR PID.

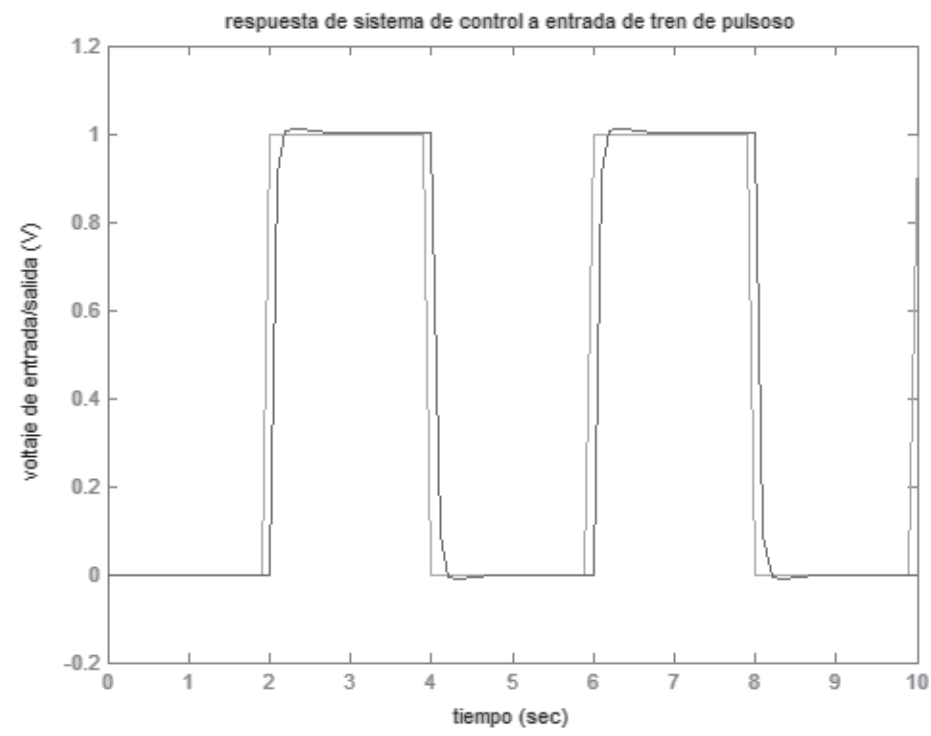

FIGURA 16. RESPUESTA DEL MOTOR GOBERNADO POR EL CONTROLADOR PI. 
Con estas pruebas finaliza el proceso de diseño de los controladores. Los sistemas de control diseñados será la base para la implementación física del control de un objeto móvil, para controlar la velocidad de los motores de corriente continua por medio del voltaje de la armadura (rotor).

\section{Implementación de la transmisión inalámbrica de datos}

Para realizar el control inalámbrico y gobernar el movimiento de un sistema electromecánico, se selecciono la tecnología ZigBee. Se trata de un protocolo para comunicaciones inalámbricas de área personal denominado WPAN (Wireless Personal Área Network). En el mercado están disponibles distintos radio transreceptores y se seleccionaron módulos Xbee Serie 2. El protocolo ZigBee permite establecer transmisión inalámbrica de datos entre dos o más dispositivos a corta distancia; los dispositivos tienen bajo consumo de energía, baja tasa de transmisión de datos y son de bajo costo ${ }^{[22]}$. Los módulos Xbee se configuraron para la comunicación punto a punto, utilizando el software libre suministrado por el fabricante Digi International. La tecnología ZigBee es satisfactoria para las necesidades de este sistema debido al requerimiento de transmitir baja cantidad de datos a corta distancia.

\section{Conclusión}

En este artículo se presentó el proceso de modelado matemático y la identificación de un motor de corriente continua con imán permanente. Fue muy importante el uso de las herramientas computacionales para verificar las funciones de transferencia obtenidas y para el diseño de sistema de control. Se utilizó el software de Matlab con las herramientas que ofrece (Simulink, Simscape, System Identification Tool y SISO Tool). En el proceso de diseño del control se utilizaron ambas funciones de transferencia y se presentaron los resultados del diseño de dos tipos de controladores PID y PI. Según la simulación realizada en el software de Matlab ambos sistemas de control responden satisfactoriamente. En la siguiente etapa está prevista la implementación física de sistema de control PID diseñada.

Los resultados obtenidos se van a utilizar como parte necesaria para construir el control de un objeto móvil, donde se requiere gobernar inalámbri- 
camente su desplazamiento de una manera muy precisa. En el proceso de implementación de sistema de control se van a utilizar tecnologías de fácil implementación: la tarjeta de Arduino UNO con la tarjeta Arduino Motor Shield y Arduino XBee Shield (para la implementación de control de los motores) y la tarjeta de Arduino UNO con la tarjeta Arduino XBee Shield y Arduino Joystick Shield (para la implementación de comandos de mando). Se seleccionó la tecnología de Arduino y módulos Xbee por su bajo costo, fácil interconexión y las características técnicas satisfactorias para este proyecto.

\section{Referencias}

[1] K. Ogata, “Ingeniería de Control Moderno”, Prentice Hall, Madrid, 2010.

[2] R. Dorf, R. Bishop, “Sistemas de Control Moderno", Prentice Hall, Madrid, 2005.

[3] N. S. Nise. "Control systems engineering”, John Wiley \& Sons, Jefferson City, 2011.

[4] V. M. Hernández, R. Silva, R. V. Carrillo Serrano, "Control Automático”, Colección CIDETEC, México, 2013. [Online available: http://www.controlautomatico.com.mx/ book/CONTROL\%2OAUTOMATICO\%202013_HIGH.pdf].

[5] F. X. Blasco, M. A. Martínez, J. S. Senent, J. Sanchis, "Sistemas Automáticos”, Universidad Politécnica de Valencia, Valencia, 2000.

[6] M. G. Quijano, C. G. Hernández, “Obtención experimental de los parámetros del motor que se utilizará en el sistema de locomoción de una esfera rodante”, Tesis de la Universidad Pontificia Bolivariana, Colombia, 2009.

[7] S. E. Toledo, "Diseño de controladores PID en tiempo discreto y análisis de respuesta utilizando herramientas computacionales", Tesis de la Universidad de San Carlos de Guatemala, Guatemala, 2007.

[8] MathWorks, “Simulink User's Guide”, The MathWorks Inc., Natick, 2015.

[9] MathWorks, “Simscape User's Guide”, The MathWorks Inc., Natick, 2014.

[10] L. Ljung. "System Identification Toolbox - Getting Started Guide", The MathWorks Inc., Natick, 2015.

[11] C. Urrea, J. Kern, “Characterization, Simulation and Implementation of a New Dynamic Model for a DC Servomotor”, IEEE Latin America Transactions, Vol. 12, No. 6, pp. 997-1004, September 2014.

[12] M. S. Álvarez, "Modelo Matemático de un Motor de Corriente Continua Separadamente Excitado: Control de Velocidad por Corriente de Armadura", Latin American Journal of Physics Education, Vol. 6, No. 1, pp. 155-161, March 2012. 
[13] Pololu Robotics\&Electronics, “Metal Gearmotor 30:1". [Online available: https:// www.pololu.com/product/1103].

[14] MathWorks, “Arduino Support from MATLAB”, The MathWorks, Inc., [Online available: http://www.mathworks.com/hardware-support/arduino-matlab.html].

[15] M. Fruk, G. Vujisic, T. Spoljaric, "Parameter Identification of Transfer Functions Using MATLAB", 36th International Convention on Information \& Communication Technology Electronics \& Microelectronics (MIPRO), pp. 697-702, 2013.

[16] D. A. Zacarias, "Estudio y Control Automático Retroalimentado de un Motor de CD de Laboratorio con las Herramientas de Matlab y Labview" (Tesis), Universidad Veracruzana, Xalapa, 2011.

[17] J. D. Rairán, C. E. Guerrero y J. A. Mateus, “Diseño de Controladores de Tipo Proporcional Integral Derivativo (PID) y Difuso para la Posición de un Motor de Corriente Continua (DC)", Revistas Científicas Pontificia Universidad Javeriana - Ingeniería y Universidad, Vol. 14, No.1, pp. 137-160, Enero-Junio de 2010. [Online available: http:// revistas.javeriana.edu.co/index.php/iyu/article/view/972/570].

[18] E. Rodríguez, J. Páez, "Diseño Mecatrónico de una Shield de Arduino para el Control de Motores DC con Escobillas”, Prospect, Vol. 14, No. 1, pp. 73-79, Enero-Junio 2016. [Online available: http://ojs.uac.edu.co/index.php/prospectiva/article/view/370/ pdf_32].

[19] P. Vikhe, N. Punhabi, C. Kadu, “Real Time DC Motor Speed Control using PID Controller in Labview”, International Journal of Advanced Research in Electrical, Electronics and Instrumentation Engineering, Vol. 3, Issue 9, Sep. 2014.

[20] N. Korenic, M. Horvatic, “Upravljanje Brzinom Vrtnje Malog Istosmjernog Elektromotora Koristenjem Arduino Razvojne Platforme", Technical Jornal, Vol. 9, No. 1, pp 70-76, Sep. 2015. [Online available: http://hrcak.srce.hr/index.php?show=clanak\&id clanak_jezik=203363].

[21] P. Vinnacota, "Motor Control with Arduino: A Case Study in Data-Driven Modeling and Control Design", The MathWorks, Inc., 2013. [Online available: http://www.mathworks.com/tagteam/77442_92066voo_motor-control-with-arduino-a-case-studyin-design.pdf]

[22] J. Titus, “The Hands-on Xbee Lab Manual”, Elsevier, Waltham, 2012. 